\title{
Kinetic mixing as the origin of a light dark-gauge-group scale
}

\author{
Clifford Cheung, ${ }^{1,2}$ Joshua T. Ruderman, ${ }^{3}$ Lian-Tao Wang, ${ }^{3}$ and Itay Yavin ${ }^{3}$ \\ ${ }^{1}$ School of Natural Sciences, Institute for Advanced Study, Princeton, New Jersey 08540, USA \\ ${ }^{2}$ Department of Physics, Harvard University, Cambridge, Massachusetts 02138, USA \\ ${ }^{3}$ Department of Physics, Princeton University, Princeton, New Jersey 08544, USA
}

(Received 2 March 2009; published 12 August 2009)

\begin{abstract}
We propose a model in which supersymmetric weak scale dark matter is charged under a $U(1)_{d}$ dark gauge symmetry. Kinetic mixing between $U(1)_{d}$ and hypercharge generates the appropriate hierarchy of scales needed to explain PAMELA and ATIC with a GeV scale force carrier and DAMA (or INTEGRAL) using the proposals of inelastic (or, respectively, exciting) dark matter. Because of the extreme simplicity of this setup, observational constraints lead to unambiguous determination of the model parameters. In particular, the DAMA scattering cross section is directly related to the size of the hypercharge D-term vacuum expectation value. The known relic abundance of dark matter can be used to fix the ratio of the dark sector coupling to the dark matter mass. Finally, the recent observation of cosmic ray positron and electron excesses can be used to fix the mass of the dark matter through the observation of a shoulder in the spectrum and the size of the kinetic mixing by fitting to the rate. These parameters can be used to make further predictions, which can be checked at future direct detection, indirect detection, as well as collider experiments.
\end{abstract}

DOI: 10.1103/PhysRevD.80.035008

PACS numbers: 12.60.Jv, 12.60.Cn, 12.60.Fr

\section{INTRODUCTION}

A number of intriguing results from astronomical and cosmic ray data may be evidence for dark matter (DM) annihilation in our galaxy [1,2]. In addition, the DAMA direct detection experiment reports a signal which may be the first instance of DM interaction with normal matter [3]. Interestingly, if interpreted as coming from dark matter interactions and annihilations, these signals span an enormous hierarchy of length scales, $100 \mathrm{keV}-1 \mathrm{TeV}$, making dark matter model building a challenging enterprise. Along these lines, Arkani-Hamed et al. have suggested a broad framework [4] in which 500-800 GeV dark matter is charged under a dark gauge group, $G_{d} \supset U(1)_{d}$, whose Abelian factor kinetically mixes with the standard model (SM) photon [5]. Assuming that $G_{d}$ is non-Abelian and broken at a $\mathrm{GeV}$, then loops of the resulting $\mathrm{GeV}$ scale dark gauge bosons will generate $100 \mathrm{keV}-1 \mathrm{MeV}$ mass splittings within the dark matter multiplet.

This approach resolves several of the puzzles raised by recent observations: (1) A Sommerfeld enhancement from $\mathrm{GeV}$ scale dark gauge bosons boosts the dark matter annihilation rate today, reconciling the large flux of $e^{+} e^{-}$ observed in the PAMELA and ATIC data with the weak cross section inferred from the dark matter relic abundance. (2) Dark gauge bosons have kinematically suppressed hadronic decays, explaining the lack of excess antiprotons at PAMELA. (3) The DAMA signal arises from inelastic nuclei-dark matter (iDM) scattering coming from $\sim 100 \mathrm{keV}$ mass splittings in the dark matter multiplet [6]. (4) A slightly larger splitting of $\sim 1 \mathrm{MeV}$ can explain the INTEGRAL $511 \mathrm{keV}$ line, using the proposal of exciting dark matter $[7,8]$.
In this paper, we propose a simple and predictive setup that differs from the original proposal in two important ways. First, we demonstrate that an Abelian gauge group, $G_{d}=U(1)_{d}$, is sufficient to generate a multiplet of states with $100 \mathrm{keV}-1 \mathrm{MeV}$ mass splittings. This a considerable simplification over non-Abelian models, which generally need large numbers of dark Higgses [9]. Second, we argue that supersymmetric theories acquire a scale of $\sim \mathrm{GeV}$ as a dynamical consequence of kinetic mixing alone. D-term mixing between $U(1)_{d}$ and the minimal supersymmetric SM (MSSM) hypercharge induces an effective FayetIliopoulos (FI) term for $U(1)_{d}$ of order $\mathrm{GeV}^{2}$. In the presence of a single $U(1)_{d}$ charged dark Higgs, the dark gauge symmetry is broken at a $\mathrm{GeV}$.

Our model is also distinct from a number of alternative proposals for $U(1)_{d}$ charged dark matter. An earlier treatment has neglected D-term mixing completely, even though it is dominant to the effects being considered [10]. Another approach considers gauge mediation in the limit of negligible kinetic mixing [11]. Also, during the completion of this work we learned of an interesting forthcoming proposal [12] which utilizes anomaly mediation to generate additional $\mathrm{GeV}$ scale contributions.

The model presented in this paper is remarkably simple and has a small number of free parameters that can be fixed by observations. For instance, the dark matter mass, $M$, may be fixed by a possible future observation of a shoulder in the PAMELA positron excess (alternatively, the feature seen in ATIC may already fix that scale). Assuming thermal freeze-out, the dark gauge coupling, $g_{d}$, determines the relic abundance and is thus fixed in terms of $M$. Furthermore, the size of the kinetic mixing, $\epsilon$, is constrained by the boost factor required to explain the PAMELA positron 
excess. Quite interestingly, our model implies a direct relation between the DAMA scattering cross section and the hypercharge D-term of the MSSM. This is a generic prediction of any theory whose only origin of scales is the kinetic mixing. Also, the dark photon should be copiously produced in supersymmetry (SUSY) cascades at particle colliders, yielding clusters of closely packed collimated energetic leptons, which has been referred to in Refs. [4,9] as "lepton jets." At high energy colliders, dark photons are produced with energies of tens to hundreds GeV. As the dark photon decays through its mixing with the photon it produces a pair of leptons that are highly collimated $\Delta R_{\ell \ell} \sim 0.1-0.01$, forming lepton jets. Cascade decays in the $\mathrm{GeV}$ dark sector will result in more leptons and, hence, richer lepton jets, see [9] for a detailed discussion. We note that the dark photon can also have significant decay branching ratios to light mesons. Presence of such mesons certainly alters the phenomenology, and it is therefore possible to define several subclasses of lepton jets. Since our focus is not on the collider phenomenology, we will not make this distinction in this paper.

\section{NEW SCALES IN THE DARK SECTOR VIA KINETIC MIXING}

Throughout this paper we assume a weak scale mass for the dark matter supermultiplet. All of the light scales in the dark sector will be generated dynamically through kinetic mixing between hypercharge and the dark force carrier. In particular, the action contains a term

$$
\mathcal{L} \supset-\frac{\epsilon}{2} \int d^{2} \theta W_{Y} W_{d},
$$

where $W_{Y}\left(W_{d}\right)$ is the supersymmetric field strength for the SM hypercharge (dark Abelian group) and $\epsilon$ is a small parameter. Integrating out heavy fields charged under both hypercharge and $U(1)_{d}$ will induce this operator and we can estimate the size of the mixing to be

$$
\epsilon=-\frac{g_{Y} g_{y}}{16 \pi^{2}} \sum_{i} Q_{i} q_{i} \log \left(\frac{M_{i}^{2}}{\mu^{2}}\right),
$$

which can naturally be of order $10^{-3}-10^{-4}$. As observed in Ref. [9], the kinetic mixing includes

$$
V_{\text {D-term mixing }}=\epsilon D_{Y} D_{d} \text {. }
$$

After electroweak symmetry breaking, $D_{Y}$ gets a vacuum expectation value (VEV)

$$
\left\langle D_{Y}\right\rangle=\frac{g^{\prime}}{2}\left(\left|H_{u}\right|^{2}-\left|H_{d}\right|^{2}\right)+\xi_{Y},
$$

where $H_{u, d}$ are the MSSM Higgs doublets and $g^{\prime}$ is the hypercharge coupling. We included $\xi_{Y}$, which is an effective FI term for the SM hypercharge group, since this is a relevant operator allowed by all the symmetries and there is no reason to a priori exclude it from the low energy action that defines the MSSM [13-15]. From the low energy perspective, $\xi_{Y}$ is only constrained to not be so large as to destabilize the electroweak scale.

The expectation value of $D_{Y}$ induces an effective FI term for the dark Abelian group via the kinetic mixing

$$
\xi=\epsilon\left\langle D_{Y}\right\rangle=\epsilon\left(-\frac{g^{\prime} v^{2} \cos 2 \beta}{4}+\xi_{Y}\right),
$$

where $v$ is the electroweak vacuum expectation value. With $\epsilon=10^{-4}-10^{-3}$ and $\left\langle D_{Y}\right\rangle$ of order the weak scale, we find that $\xi=(1-5 \mathrm{GeV})^{2}$. Thus the $\mathrm{GeV}$ scale in the dark sector is a fortuitous by-product of the kinetic mixing. If there are any light degrees of freedom charged under $U(1)_{d}$, the vacuum can break the gauge group and/or supersymmetry at the GeV scale. We focus on the possibility that SUSY is preserved and $U(1)_{d}$ is broken, resulting in a $\sim \mathrm{GeV}$ mass for the vector supermultiplet. If dark matter has a superpotential coupling to a light field that gets a VEV, then the vacuum can dynamically generate a $\mathrm{MeV}$ sized splitting between dark matter supermultiplets, as we now demonstrate with a concrete model.

\section{A MODEL FOR THE DARK SECTOR}

We take DM to be a pair of chiral supermultiplets, $\left(\Phi, \Phi^{c}\right)$, oppositely charged under the dark gauge group, $U(1)_{d}$. The superpotential for chiral multiplets is given by

$$
W=M \Phi \Phi^{c}+\lambda N h h^{c}+\frac{1}{4 \Lambda} \Phi^{2} h^{c 2},
$$

$h$ and $h^{c}$ are oppositely charged and $N$ is neutral under $U(1)_{d}$. Here $\Lambda \sim \mathrm{TeV}$ and is associated with new physics at the electroweak scale [16]. We choose a discrete symmetry to forbid dimensionful operators involving the light fields: $N, N^{2}$, and $h h^{c}$. Depending on the choice of discrete symmetry, there may be marginal and irrelevant operators in addition to the ones included in Eq. (6), but they will not be relevant to the following discussion. The neutral field is included in order to avoid any massless degrees of freedom at low energies.

The scalar potential for this theory is given by:

$$
\begin{aligned}
V= & V_{D}+V_{F} \\
V_{D}= & \frac{1}{2}\left[\frac{g_{d}}{2}\left(|h|^{2}-\left|h^{c}\right|^{2}+|\Phi|^{2}-\left|\Phi^{c}\right|^{2}\right)+\xi\right]^{2} \\
V_{F}= & \left|M \Phi^{c}+\frac{1}{2 \Lambda} \Phi h^{c 2}\right|^{2}+|M \Phi|^{2} \\
& +\left|\lambda N h+\frac{1}{2 \Lambda} h^{c} \Phi^{2}\right|^{2}+\left|\lambda N h^{c}\right|^{2}+\left|\lambda h h^{c}\right|^{2} .
\end{aligned}
$$

There is a SUSY vacuum (with vanishing F and D-terms) with broken $U(1)_{d}$ at $\left\langle h^{c}\right\rangle=\sqrt{\frac{2 \xi}{g_{d}}}$ and all other scalar VEVs set to zero. 


\section{A. Coupling to the standard model}

The supersymmetric field strength mixing, Eq. (1), also contains the gauge-boson kinetic mixing

$$
\mathcal{L}_{\text {gauge mixing }}=\frac{\epsilon}{2} B_{\mu \nu} b^{\mu \nu},
$$

where $B_{\mu \nu}$ and $b_{\mu \nu}$ denote the SM and dark field strengths. As argued in [4], this is the leading marginal operator that couples the dark sector to the standard model. Moreover, since it does not violate any SM symmetries, it is relatively unconstrained phenomenologically, and $\epsilon=10^{-4}-10^{-3}$ is consistent with current bounds [17].

The primary effect of this mixing is to induce an $\epsilon$ suppressed coupling between the electromagnetic current of the SM and the dark vector-boson [18]. Dark matter annihilations produce dark vector-bosons that subsequently decay into light leptons or pions via this kinetic mixing. Decay into heavier particles is kinematically disallowed. This injection of leptons can explain the excesses observed at PAMELA and ATIC. Another consequence of the kinetic mixing is that the SM $Z$ boson and bino couple to the $U(1)_{d}$ current, which has implications for collider phenomenology [9].

\section{B. Mass spectrum}

Next, let us consider the spectrum of the dark sector. The vector supermultiplet gets a mass of

$$
m_{b}^{2}=g_{d} \xi
$$

which is naturally $\sim \mathrm{GeV}$ scale. As pointed out in Refs. $[4,8]$, a vector-boson of this mass elegantly explains why decay channels into antiprotons are kinematically disfavored. It also serves as a light force carrier capable of enhancing the annihilation cross section via the Sommerfeld enhancement mechanism to produce the necessary annihilation rate for PAMELA or ATIC.

The masses of the dark matter states $\Phi$ and $\Phi^{c}$ are affected as well. To leading order in $m_{b} / M \lessgtr 10^{-3}$, the mass eigenstates are given by $\Phi_{ \pm}=\left(\Phi \pm \Phi^{c}\right) / \sqrt{2}$, and $\Phi_{-}$, being the lighter of the two, is identified with our (fully supersymmetric) dark matter candidate. The mass splitting between the two states is

$$
\begin{aligned}
\Delta m & =m_{+}-m_{-}=\frac{m_{b}^{2}}{g_{d}^{2} \Lambda} \\
& =0.8 \mathrm{MeV}\left(\frac{30^{-1}}{\alpha_{d}}\right)\left(\frac{m_{b}}{\mathrm{GeV}}\right)^{2}\left(\frac{3 \mathrm{TeV}}{\Lambda}\right) .
\end{aligned}
$$

Depending on the precise values of the parameters involved, this scale can be used to explain either DAMA or INTEGRAL using the iDM or exciting dark matter scenarios, respectively. Either way, the mass splitting can be fixed by fitting to the experimental signature. Furthermore, since the states are almost maximally mixed, the transitions among mass eigenstates are strongly inelastic. The elastic couplings are suppressed by $m_{b}^{2} / M \Lambda$, and bounds from direct detection are easily evaded. If $\Delta m<2 m_{\text {electron }}$, then the lifetime of the excited state is longer than the age of the Universe [19]. Direct detection bounds may be relevant if a cosmological abundance of the excited state is still present today.

Lastly, we consider the spectrum of dark Higgses. $h^{c}$ is eaten via the super-Higgs mechanism. $h$ and $N$ pair-up and become massive:

$$
m_{h}^{2}=m_{N}^{2}=\frac{2 \lambda^{2} \xi}{g_{d}}=2\left(\frac{\lambda}{g_{d}}\right)^{2} m_{b}^{2} .
$$

As long as $\lambda>g_{d} / 2 \sqrt{2}$, then $m_{h}>m_{b} / 2$ and decays of the dark photon into dark Higgses are kinematically forbidden. At this level, these states are exactly stable since the potential respects $N-h$ number as an exact symmetry.

\section{Supersymmetry breaking effects}

This setup is interesting because the origin of scales is centered on the mixed D-term. For this reason, we have assumed there are no large SUSY breaking effects, for example, from gravity mediation. Instead, we consider low-scale gauge mediation such that the dark sector only receives SUSY breaking contributions from the MSSM via kinetic mixing. As such, the dark sector is supersymmetric only at leading order in $\epsilon$. For example, since the dark Higgs couples to the MSSM bino, it receives a positive soft mass of order $m^{2} \sim \epsilon^{2} g_{d}^{2} M_{\tilde{B}}^{2} / 16 \pi^{2} \sim(10-100 \mathrm{MeV})^{2}$ from bino loops. This has a negligibly small effect on the dark matter supermultiplet, since it lifts the scalars from the fermions by $m^{2} / M \sim \mathcal{O}(1) \mathrm{keV}$. The dark matter is effectively supersymmetric because transitions among the supermultiplet occur on time scales longer than the age of the Universe.

In contrast, the scalar $h$ is heavier than its superpartner by an amount $m^{2} / m_{h} \sim \mathcal{O}(1) \mathrm{MeV}$, while the scalar $N$ is lighter by a tenth of that due to a negative mass ${ }^{2}$ contribution from the Yukawa coupling, $\lambda N h h^{c}$. Furthermore, an A-term of size $\lambda \epsilon^{2} g^{\prime 2} M_{\tilde{B}} / 16 \pi^{2}$ is generated which mixes $N$ and $h$ once $h^{c}$ condenses. The resulting spectrum consists of the scalar $N$ as the lightest state, the fermionic $N$ and $h$ about $100 \mathrm{keV}-\mathrm{MeV}$ above that, and the scalar $h$ as the heaviest state. While these MeV splittings imply interesting possibilities for model building, we leave this for future work.

\section{OBSERVATIONS AND PREDICTIONS}

In this section, we discuss the parameters of our model and their relation to observations in astrophysics and direct detection.

The mass of the weakly interacting massive particle (WIMP) can be determined through the electron/positron excess seen in ATIC/PAMELA. Since the leptons are produced as by-products of the annihilation into dark 
photons, $\Phi \Phi \rightarrow \gamma^{\prime} \gamma^{\prime}$, followed by a leptonic decay of $\gamma^{\prime}$, the excess should have an endpoint at the WIMP's mass.

The relic abundance of dark matter is fixed by the thermal annihilation cross section of $\Phi$ during freeze-out. The dominant annihilation channel is $\Phi \Phi \rightarrow \gamma^{\prime} \gamma^{\prime}$, which fixes the dark gauge coupling in terms of the dark matter mass [20]:

$$
\langle\sigma v\rangle_{\text {freeze }} \sim \alpha_{d}^{2} / M^{2},
$$

with $\langle\sigma v\rangle_{\text {freeze }}=3 \times 10^{-26} \mathrm{~cm}^{3} \mathrm{~s}^{-1}$.

Next, let us consider the effects of the $\mathrm{GeV}$ scale states on cosmology. Although $N$ and $h$ are stable, their relic abundance can be sufficiently depleted assuming that they are heavier than the vector multiplet. In this case, they annihilate efficiently into the vector multiplet, which, in turn, annihilates into SM fields via the kinetic mixing. Since this cross section is small, the states in the vector multiplet have large abundances at freeze-out, but all decay safely before big bang nucleosynthesis (BBN). In particular, the dark photon decays promptly to $e^{+} e^{-}$, while the radial dark Higgs decays either into $e^{+} e^{-}$through a loop or into $e^{+} e^{-} e^{+} e^{-}$through two off shell dark photons. The dark photino has the longest lifetime because it has to decay to a photon and gravitino. This decay can occur before BBN:

$$
\tau_{\tilde{\gamma}^{\prime} \rightarrow \gamma \tilde{G}} \sim 0.3 \mathrm{~s}\left(\frac{10^{-3}}{\epsilon}\right)^{2}\left(\frac{\mathrm{GeV}}{m_{b}}\right)^{5}\left(\frac{\sqrt{F}}{10 \mathrm{TeV}}\right)^{4} .
$$

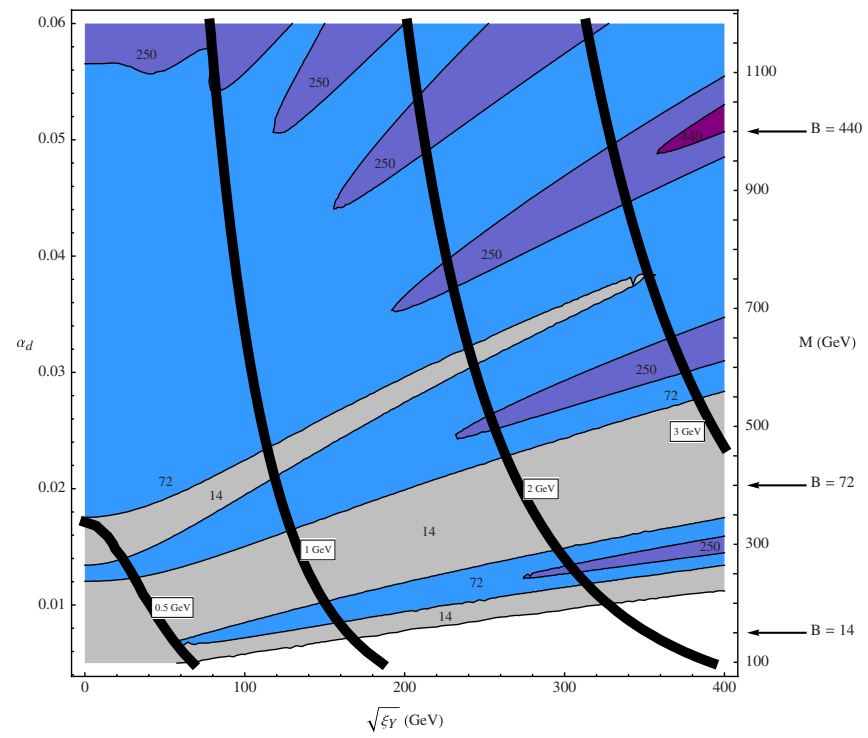

FIG. 1 (color online). A contour plot of the Sommerfeld enhancement [4] as a function of $M$ [or $\alpha_{d}$, related through Eq. (12)] and $\sqrt{\xi_{Y}}$, with $\epsilon=10^{-4}$ and $\tan \beta=40$. The solid black lines correspond to fixed $m_{b}$. The dark matter velocity is taken to be $v=150 \mathrm{~km} / \mathrm{s}$. On the right we indicate the boost required for PAMELA for three reference values of $M$, for the process $\Phi \Phi \rightarrow \gamma^{\prime} \gamma^{\prime}$ followed by $\gamma^{\prime} \rightarrow e^{+} e^{-}$[23]. The boost is relative to $\langle\sigma v\rangle=3 \times 10^{-26} \mathrm{~cm}^{3} \mathrm{~s}^{-1}$, assuming local dark matter density $\rho_{0}=0.3 \mathrm{GeV} \mathrm{cm}^{-3}$.

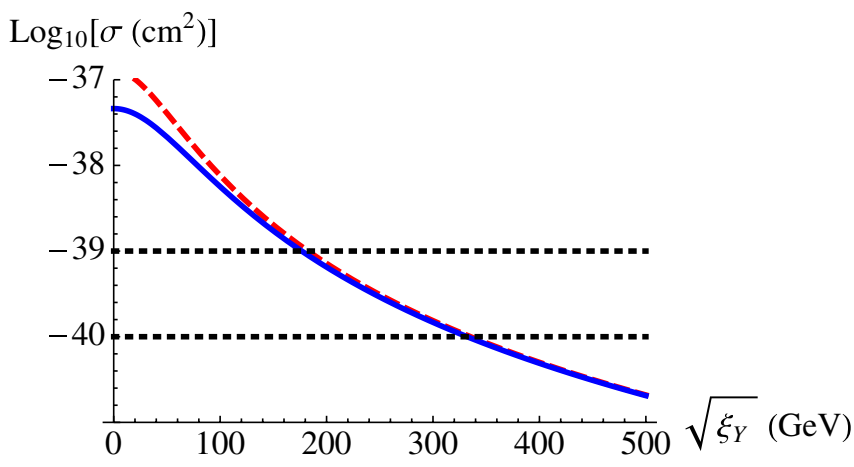

FIG. 2 (color online). The DAMA cross section as a function of the effective FI parameter with $\tan \beta=2$ (red, dashed line) and $\infty$ (blue, line). The horizontal lines indicate the preferred range for the WIMP-nucleon cross section [6].

The dark photon $\gamma^{\prime}$ provides the Sommerfeld enhancement of the annihilation cross section needed for PAMELA [1] and ATIC [2]. In Fig. 1, we present a contour plot of the Sommerfeld enhancement $S$ as a function of $\xi_{Y}$ and $M$.

Our model can reconcile DAMA with the limits of other direct detection experiments, if $\Lambda$ is such that the mass splitting between $\Phi_{+}$and $\Phi_{-}$is $\Delta m \sim 100 \mathrm{keV}$, providing a realization of the iDM scenario of Ref. [6]. Interestingly enough, the cross section per nucleon in this model is sensitive to $\left\langle D_{Y}\right\rangle$ only,

$$
\sigma=\frac{4 Z^{2} \mu_{n e}^{2}}{A^{2}} \frac{\alpha \cos ^{2} \theta_{W}}{\left\langle D_{Y}\right\rangle^{2}} .
$$

Notice that any dependence on the dark sector couplings have cancelled completely. This type of cancelation will occur in any theory in which the mass scale of the dark sector is fixed entirely by D-term kinetic mixing. Thus, for this class of models the measured DAMA cross section, if confirmed, yields a definitive prediction about electroweak physics. Figure 2 shows the dependence of the DAMA cross section on $\xi_{Y}$, where we have also denoted the range of cross sections preferred by iDM [6].

A determination of $\xi$ and $\alpha_{d}$ yields a prediction for $m_{b}$, the mass of the vector supermultiplet, as shown in Fig. 1. Furthermore, since the MSSM bino couples directly to the dark photon and photino, dark photons should be produced in SUSY cascades from the MSSM at high energy colliders. Such a signal, very distinct from the collider signatures of the conventional MSSM, is generic in GeV dark sector models with supersymmetry [9]. Dark state production at the LHC provides a promising avenue for probing the dark sector and its interactions $[9,21]$.

\section{CONCLUSIONS}

In this paper we considered a supersymmetric dark sector involving a massive and stable matter field, which constitute the WIMP and is coupled to an Abelian gauge field. The sector also contains light matter fields which 
ultimately spontaneously break the gauge symmetry at $\sim \mathrm{GeV}$. Similarly to recently proposed scenarios, the Abelian group is weakly mixed with hypercharge through the kinetic terms. The main point of our discussion is that, in supersymmetry, the existence of kinetic mixing, together with the breaking of hypercharge in the SM, also breaks the dark Abelian gauge symmetry at $\sim \mathrm{GeV}$ in a natural and unavoidable fashion.

We find the extreme simplicity of this setup and the natural generation of all the scales involved the most attractive feature of this scenario. However, as it stands, it suffers from two main difficulties. The first is related to the lifetime of the dark gaugino given in Eq. (13). In order to decay before BBN it requires a rather low supersymmetry breaking scale. This can easily be relaxed with a slightly heavier gauge boson or larger mixing parameter. Moreover, the injection of electromagnetic energy during $\mathrm{BBN}$ is not as strongly constrained as hadronic energy injection [22]. The second issue, and the more serious one, is associated with the cross section of WIMP-nucleon scattering in the iDM model as given in Eq. (14). The model's simplicity leads to an unambiguous relation between this cross section and the MSSM hypercharge $D$-term, $\left\langle D_{Y}\right\rangle$. In the simplest case, where $\left\langle D_{Y}\right\rangle$ is given by the Higgs' VEV alone, the cross section is too large by about an order of magnitude. It is certainly possible for $\left\langle D_{Y}\right\rangle$ to enjoy from additional contributions, however, some of the model's allure is marred.

Despite these shortcomings, both of which can be alleviated with the slightest extensions, this model serves as an example for an incredibly simple dark sector which naturally generates all the necessary scales recently discussed in the literature. It exhibits a rich structure originating from the single inclusion of supersymmetric kinetic mixing between hypercharge and a dark Abelian gauge group. Most importantly, it is predictive in its content and results in unambiguous relations between its parameters and measurable quantities.

\section{ACKNOWLEDGMENTS}

We would like to thank N. Arkani-Hamed, M. Popsleov, and $\mathrm{N}$. Weiner for very useful discussions, especially in regard to the long lifetimes of the excited state discussed in the text. We would especially like to thank T. Slatyer for help in generating the Sommerfeld enhancement plot. L.-T. W. and I. Y. are supported by the National Science Foundation under grant PHY-0756966 and the Department of Energy under grant DE-FG02-90ER40542. J.T.R. is supported by the National Science Foundation .

Note added.-Several months after the completion of the initial version of this paper, Fermi-LAT published its first measurement of the $e^{+}+e^{-}$spectrum, which shows a deviation from the conventional background prediction [24]. A boost factor on the order of $10^{2}$ is still necessary to account for the excess from dark matter annihilations [25]. The model presented in this paper remains a promising candidate for this scenario.
[1] O. Adriani et al., Phys. Rev. Lett. 102, 051101 (2009); Nature (London) 458, 607 (2009).

[2] J. Chang et al., Nature (London) 456, 362 (2008).

[3] R. Bernabei et al. (DAMA Collaboration), Eur. Phys. J. C 56, 333 (2008).

[4] N. Arkani-Hamed, D. P. Finkbeiner, T. Slatyer, and N. Weiner, Phys. Rev. D 79, 015014 (2009); N. ArkaniHamed and N. Weiner, J. High Energy Phys. 12 (2008) 104.

[5] M. Pospelov, A. Ritz, and M. B. Voloshin, Phys. Lett. B 662, 53 (2008).

[6] D. Tucker-Smith and N. Weiner, Phys. Rev. D 64, 043502 (2001); S. Chang, G. D. Kribs, D. Tucker-Smith, and N. Weiner, Phys. Rev. D 79, 043513 (2009); Y. Cui, D.E. Morrissey, D. Poland, and L. Randall, J. High Energy Phys. 05 (2009) 076.

[7] D. P. Finkbeiner and N. Weiner, Phys. Rev. D 76, 083519 (2007); M. Pospelov and A. Ritz, Phys. Lett. B 651, 208 (2007).

[8] I. Cholis, L. Goodenough, and N. Weiner, Phys. Rev. D 79, 123505 (2009).

[9] M. Baumgart, C. Cheung, J. T. Ruderman, L. T. Wang, and
I. Yavin, J. High Energy Phys. 04 (2009) 014.

[10] K. M. Zurek, Phys. Rev. D 79, 115002 (2009).

[11] E. J. Chun and J. C. Park, J. Cosmol. Astropart. Phys. 02 (2009) 026

[12] A. Katz and R. Sundrum, J. High Energy Phys. 06 (2009) 003.

[13] A. de Gouvea, A. Friedland, and H. Murayama, Phys. Rev. D 59, 095008 (1999).

[14] S. Dimopoulos and G. F. Giudice, Phys. Lett. B 393, 72 (1997).

[15] I. Jack and D. R. T. Jones, Phys. Lett. B 482, 167 (2000); B. Murakami and J.D. Wells, Phys. Rev. D 68, 035006 (2003).

[16] This can come in the form of $\mathrm{TeV}$ scale states, which couple to both $\Phi$ and $h^{c}$. Then the operator $\frac{1}{4 \Lambda} \Phi^{2} h^{c 2}$ can be generated after integrating out the heavy states. One simple possibility is to introduce an additional pair of chiral supermultiplets $S, S^{c}$ with Dirac mass $\sim \mathrm{TeV}$, and couplings $\lambda_{\Phi} S \Phi^{2}+\lambda_{h^{c}} S^{c} h^{c 2}$.

[17] M. Pospelov, arXiv:0811.1030.

[18] B. Holdom, Phys. Lett. 166B, 196 (1986).

[19] See Ref. [26] for details and lifetime estimates. 
[20] We leave a more detailed calculation of the relic abundance to a future publication.

[21] Y. Bai and Z. Han, arXiv:0902.0006.

[22] K. Jedamzik, Phys. Rev. D 74, 103509 (2006).

[23] I. Cholis, G. Dobler, D. P. Finkbeiner, L. Goodenough, and N. Weiner, arXiv:0811.3641.
[24] A. A. Abdo et al. (The Fermi LAT Collaboration), Phys. Rev. Lett. 102, 181101 (2009).

[25] P. Meade, M. Papucci, A. Strumia, and T. Volansky, arXiv:0905.0480.

[26] D. P. Finkbeiner, T. R. Slatyer, N. Weiner, and I. Yavin, arXiv:0903.1037. 